\title{
Strukturierte Versorgung von Mukoviszidosepatienten und ihren Angehörigen in einem ISO-zertifizierten Behandlungszentrum
}

\author{
Structured Care in an ISO Certified Centre for Patients With Cystic Fibrosis and \\ Their Families
}

Autoren

Institute
H. Ellemunter ${ }^{1}$, J. Eder ${ }^{1}$, G. Steinkamp ${ }^{1,2}$

CF-Zentrum Innsbruck/Österreich

Klinische Forschung, Schwerin eingereicht 29.3. 2011 akzeptiert nach Revision 30. 5. 2011

\section{Bibliografie}

Dol http://dx.doi.org/ 10.1055/s-0030-1256591

Online-Publikation: 14. 7. 2011

Pneumologie 2011; 65:

615-623 @ Georg Thieme

Verlag KG Stuttgart · New York ISSN 0934-8387

\section{Korrespondenzadresse}

Prof. Dr. med.

Helmut Ellemunter

CF-Zentrum Innsbruck

Univ.-Klinik für Kinder- und

Jugendheilkunde

Anichstraße 35

6020 Innsbruck

Österreich

helmut.ellemunter@i-med.ac.at

\section{Zusammenfassung \\ $\nabla$}

Hintergrund: Die Mukoviszidose (CF) ist eine chronische, lebensverkürzende Erkrankung verschiedener Organsysteme. Fachgesellschaften empfehlen eine Betreuung in Spezialambulanzen mit multiprofessionellen Teams. Dargestellt wird die Organisation der Arbeit im CF-Zentrum der Universität Innsbruck sowie die damit erzielten Behandlungsergebnisse.

Patienten und Methoden: Die Behandlungsabläufe bei der multiprofessionellen Versorgung wurden sorgfältig ausgearbeitet und strukturiert. Seit 2006 erfolgen Zertifizierungen des CF-Zentrums nach DIN ISO 9001:2000. Die Patientendatenbank wird in der Sprechstunde und für das kontinuierliche Monitoring der Therapieergebnisse genutzt.

Ergebnisse: In 2010 waren 71 der insgesamt 148 Patienten (48\%) zwischen 18 und 56 Jahre alt. Seit 1995 hatte sich die Zahl der betreuten Patienten verdoppelt und der Anteil erwachsener Patienten verdreifacht. Dennoch blieben die Durchschnittswerte der FEV1 longitudinal stabil (im Median $>80 \%$ des Solls). Verglichen mit 18 anderen CFZentren (Projekt Benchmarking Mukoviszidose) waren die Innsbrucker FEV1-Werte günstig: 52\% der Erwachsenen hatten eine normale FEV1 $>80 \%$ des Solls und nur $23 \%$ eine FEV $1<50 \%$ des Solls.

Schlussfolgerungen: Die strukturierte, multiprofessionelle Betreuung war sowohl longitudinal als auch verglichen mit anderen Behandlungseinrichtungen mit günstigen Lungenfunktionsergebnissen assoziiert.

\section{Einleitung}

$\nabla$

Die Mukoviszidose (CF) als Störung exokriner Drüsen betrifft verschiedene Organsysteme. Hauptmanifestationen sind die chronisch obstruktive Lungenerkrankung und die Pankreasin-

\section{Abstract \\ $\nabla$}

Background: Cystic fibrosis (CF) is a chronic, lifeshortening disease of multiple organ systems. Guidelines recommend that patients should be treated in specialised CF centres with multi-professional teams. We describe the organisation of medical care at the CF centre of Innsbruck University as well as results of treatment.

Patients and methods: Procedures and delivery of multi-professional care have been elaborated and structured. Since 2006 the Centre has been repeatedly certified according to DIN ISO 9001:2000. The patient database is being used during the doctor's consultation and for the continuous monitoring of treatment results.

Results: In 2010, 71 of the 148 patients (48\%) were between 18 and 56 years old. The total number of patients has doubled and the proportion of adults tripled since 1995 . Nevertheless, median FEV1 remained stable ( $>80 \%$ of predicted) during the last 15 years. Compared with $18 \mathrm{CF}$ centres of the German Benchmarking Group, patients treated in Innsbruck had favourable FEV1 values: 52\% of adults had a normal FEV1 (>80\% pred.) and only $23 \%$ an FEV $1<50 \%$ of predicted.

Conclusions: A structured programme of multiprofessional care was associated with favourable treatment results, both longitudinally and in comparison to other $\mathrm{CF}$ centres.

suffizienz. Bei vielen Patienten kommen diverse andere Probleme hinzu, beispielsweise Nasenpolypen, Hepatopathie, CF-Diabetes, Unterernährung, Infertilität (bei den meisten Männern) oder Osteoporose. Während vor 60 Jahren nach der Erstbeschreibung der Erkrankung die meisten Be- 
Tab. 1 Routineversorgung in der Spezialambulanz nach den Europäischen Standards der Mukoviszidose-Versorgung [5] und ihre Umsetzung in Innsbruck.

\begin{tabular}{ll} 
Kriterium laut europäischen Standards & Innsbruck \\
Vorstellungsfrequenz & alle 3 Monate \\
- im Normalfall alle 1 bis 3 Monate & monatlich \\
- bei neu diagnostizierten Patienten häufiger & alle 6 Monate \\
- „leichte“ Phänotypen oder atypische CF alle 3 bis 6 Monate & ja \\
\hline Arzt und CF-Schwester sehen jeden Patienten und haben genügend Zeit für die Sprechstunde & ja \\
Alle Teammitarbeiter stehen beim Ambulanzbesuch zur Verfügung & ja \\
Diagnostik bei jedem Ambulanzbesuch & ja \\
- Anamnese und körperliche Untersuchung & ja \\
- Messen von Länge (bei Kindern) und Körpergewicht mit Eintragung der Werte auf Perzentilenkurven & ja \\
- Altersentsprechende Lungenfunktionstests & ja \\
- Mikrobiologische Untersuchung von Sputum oder Rachenabstrich & (Blutgasanalyse) \\
- Oximetrie & ja \\
\hline Erfassen und Besprechen der aktuellen Medikation mit genauer Erklärung etwaiger Veränderungen & ja \\
\hline Übermitteln der veränderten Medikation an den niedergelassenen Arzt & ja \\
\hline $\begin{array}{l}\text { Patienten mit B. cepacia complex oder MRSA werden außerhalb der Routine-Sprechstunden und in anderen } \\
\text { Behandlungszimmern gesehen }\end{array}$ & ja \\
\hline Es gibt separate Sprechstunden für Patienten mit und ohne P. aeruginosa-Infektion & ja \\
\hline Eine stationäre Aufnahme oder der Beginn einer ambulanten pseudomonaswirksamen Therapie mit intravenösen & ja \\
Antibiotika ist innerhalb von 24 - 48 Stunden möglich, wenn erforderlich & ja \\
\hline Im Notfall können Patienten die Spezialambulanz täglich über 24 Stunden telefonisch oder direkt erreichen & \\
\hline Für andere Anfragen gibt es eine Telefonsprechstunde & \\
\hline
\end{tabular}

troffenen bereits im Kleinkindalter verstarben, liegt heutzutage die mediane Überlebenszeit bei 35 bis 40 Jahren [1]. In 2009 waren in Deutschland und Österreich $51 \%$ bzw. 27\% der in Spezialambulanzen behandelten Patienten älter als 18 Jahre [2,3].

Ärzte und medizinisches Personal stehen vor einer Vielzahl unterschiedlicher Aufgaben, wenn sie Mukoviszidosepatienten umfassend betreuen möchten. Diese Anforderungen lassen sich am besten in multiprofessionellen Behandlungsteams bewältigen [4]. Folgerichtig hat die Europäische Mukoviszidose-Gesellschaft ECFS in ihren Behandlungsstandards multidisziplinäre Teams vorgesehen [5], und auch der Gemeinsame Bundesausschuss GbA in Deutschland erwartet diese Versorgungsform für die Kostenerstattung nach Paragraph 116 b.

An der Kinderklinik der Universität Innsbruck entstand die Spezialambulanz für Mukoviszidose in der jetzigen Struktur im Jahr 1988. Seither wurden die Behandlungsabläufe sorgfältig ausgearbeitet und strukturiert schriftlich dargestellt. Schließlich erfolgte im Jahr 2006 erstmals eine Zertifizierung des Zentrums nach DIN ISO 9001:2000. Re-Zertifizierungen wurden alle zwei Jahre ausgestellt, zuletzt 2008 nach DIN ISO 9001:2008. Ferner wurde das Zentrum von der Deutschen Gesellschaft für Pneumologie und Beatmungsmedizin (DGP) als zertifizierte CF-Einrichtung für alle Altersklassen (Kinder und Erwachsene) anerkannt.

Ziel der Arbeit ist, die wesentlichen Aspekte der strukturierten Patientenversorgung zu erläutern, wie sie sich in Innsbruck seit mehreren Jahren bewährt haben. Dabei wird der Fokus auf Schwerpunkte und Besonderheiten in diesem Zentrum gelegt, während die allgemein übliche Routine-Versorgung nicht Gegenstand dieses Aufsatzes sein soll. Dargestellt werden Strukturen und Prozesse der interdisziplinären Arbeit. Zusätzlich werden exemplarische Behandlungsergebnisse vorgestellt, auch im Vergleich zu anderen Einrichtungen.

\section{Prinzipien der Betreuung $\nabla$}

Ambulanztermine werden klar organisiert

Abgesehen von Säuglingen und Kleinkindern unter 2 Jahren, die monatlich gesehen werden, kommen die Patienten normalerweise alle drei Monate in die Ambulanz ( Tab. 1). Dabei werden Patienten aller Altersgruppen, also auch Erwachsene, im Zentrum gesehen. Während des Kalenderjahres werden in jeweils sechs Wochen eines Quartals Routine-Sprechstunden in einem „Block“ abgehalten. In diesen Phasen ist montags bis freitags von 8:00 Uhr bis 16:30 Uhr Sprechstunde. Das Behandlungsteam bereitet sich systematisch auf die Ambulanzbesuche vor und sorgt dafür, dass Diagnostik und Therapie nach einem vereinbarten Plan durchgeführt werden. Für aufwendigere Untersuchungen werden im Rahmen der Block-Termine Zeitfenster freigehalten. Auch spezielle Laborkapazitäten werden den Block-Sprechstunden zugeordnet, beispielsweise zur Messung der Fettsäuren oder der fettlöslichen Vitamine. So kann trotz einer komplexen Betreuung nichts Wichtiges vergessen werden.

Außerhalb der Blockphasen finden Sprechstunden an zweieinhalb Tagen in der Woche statt. Hier konzentriert man sich auf Besonderheiten und hat mehr Zeit, mit Patienten Gespräche außerhalb der Routine zu führen. Ambulante i.v.-Therapien mit pseudomonaswirksamen Antibiotika werden vorzugsweise in diesen Phasen organisiert.

\section{Regeln der Krankenhaushygiene werden befolgt}

Mitarbeiter des CF-Zentrums haben klare Hygiene-Richtlinien für das Personal ebenso wie für Patienten ausgearbeitet. Die CFrelevanten Bakterien, insbesondere Pseudomonaden und $B$. cepacia complex, wurden hierarchisch in eine Reihenfolge von harmlos bis gefährlich gruppiert. Die Einbestellung der Patienten zur Sprechstunde erfolgt so, dass bestimmte Tage und Räumlichkeiten Patienten mit bestimmten mikrobiologischen Befunden vorbehalten sind. Auch die Physiotherapie und andere Bereiche orientieren sich organisatorisch an der Keimsituation der Patienten. 
Tab. 2 Besonderheiten der Versorgung in Innsbruck, die über die Anforderungen der Europäischen Standards [5] hinausgehen.

Die Patientendatenbank ...

umfasst Befunde aller Arztkontakte seit 1999

dient in der Sprechstunde als Instrument zur Visualisierung der

aktuellen Situation

unterstützt bei der individuellen Therapieplanung

dient dem Zentrum zur Erfolgskontrolle der eigenen Behandlungsergebnisse

Das Arzt-Patient-Gespräch ...

umfasst die individuelle Vereinbarung von Therapiezielen

spricht dem Patienten eine aktive Rolle zu

beinhaltet strukturierte Patientengespräche bei Erreichen entwicklungspsychologisch begründeter Meilensteine (z. B. Jugendoder Partner-Gespräch)

Qualitätsmanagement...

drückt sich aus in der ISO-Zertifizierung der Ambulanz

wird im Alltag praktiziert

beinhaltet das Überprüfen der Routinen und strebt die weitere

Verbesserung der Abläufe an

Routine-Diagnostik wird systematisch ergänzt durch ..

einmal jährliche Ultra-low-dose-CTs der Lunge

moderne Lungenfunktionsdiagnostik ergänzend zur Spirometrie

(Multiple breath washout-Methode mit Messung des Lung

Clearance Index)

patientenorientierte Behandlungsergebnisse (patient reported

outcomes, PROs)

\section{Die Patientendatenbank stellt den Verlauf übersichtlich dar}

Bei jedem Ambulanzbesuch und bei jedem stationären Aufenthalt werden die Untersuchungsergebnisse des Patienten ebenso wie Anamnese und körperliche Untersuchung in die ambulanzeigene Datenbank eingegeben ( $\bullet$ Tab. 2). Sie wurde im Jahr 1999 entwickelt und vom Oberarzt des CF-Zentrums (JE) als SPSS-Datei programmiert. Die Datenbank wird fortwährend neuen Erfordernissen angepasst.

Eintragungen erfolgen direkt während der Sprechstunde durch Arzt und Schwester. Weitere Befunde werden mit Unterstützung von Dokumentationsassistenten dokumentiert. Eine Schnittstelle zum KIS-System ermöglicht die Übernahme einiger der dort erfassten, für die Versorgung relevanten Befunde.

Speziell voreingestellte Abfragen erlauben grafische und tabellarische Darstellungen des Verlaufs eines einzelnen Patienten über die Zeit. Außerdem werden für jeden Patienten die individuellen Differenzen bestimmter Parameter vom Vorjahr berechnet, beispielsweise für die FEV1 oder den Body Mass Index.

Patienten nehmen aktiv an der Behandlungsplanung teil Für den Ambulanzbesuch werden relevante Angaben in einer übersichtlichen Maske zusammengefasst ( $\bullet$ Abb. 1). Diese Informationen bilden eine wichtige Grundlage für das ärztliche Gespräch. Während der Sprechstunde betrachten die Patienten auf einem separaten Bildschirm die grafischen Verläufe und die tabellarisch zusammengefassten Laborwerte zur selben Zeit wie der Arzt.

Die Betroffenen bekommen also medizinische Kriterien zu ihrem Gesundheitszustand „in Echtzeit gespiegelt“. Dieses befähigt sie, sich selbst ein Urteil zu bilden, informierter und partnerschaftlicher mit dem Arzt zu sprechen und sich aktiver in die Planung der Betreuung einzubringen ( $\bullet$ Tab. 2).

\section{Behandlungsziele werden individuell vereinbart}

Die Situation jedes Patienten wird mit objektiven Daten dokumentiert. Zusätzlich wird das Befinden durch regelmäßig patientenorientierte Behandlungsergebnisse (patient reported outcomes, PROs) erfasst. Für die medizinische Beurteilung der Situation ist das Gesamtbild aus mehreren Parametern entscheidend. Bei der jährlichen Therapieplanungskonferenz (siehe unten) entwickelt das Behandlungsteam für jeden Patienten individuelle Behandlungsziele. Diese werden mit dem Patienten und seiner Familie durchgesprochen, nach den Wünschen und Möglichkeiten der Betroffenen modifiziert und dann für die kommenden 12 Monate zwischen Patient und Behandlungsteam vereinbart. Der schriftliche Therapieplan wird dem Patienten ausgehändigt.

\section{Besprechungen erfolgen regelmäßig mit verschiedenen Mitarbeitern}

In der wöchentlichen Teambesprechung werden alle aktuell gesehenen ambulanten Patienten diskutiert, bei denen sich Auffälligkeiten zeigen oder bei denen der Verlauf nicht ganz optimal war. An diesen Besprechungen nehmen alle Berufsgruppen teil.

Die Situation der stationären Patienten wird an jedem Wochentag im Team besprochen und jeder Patient wird täglich von einem CF-Arzt visitiert.

Alle sechs Wochen besprechen sich die CF-Ärzte mit den klinischen Mikrobiologen. Dabei werden nicht nur individuelle Patienten diskutiert, sondern es geht auch um die Infektionsepidemiologie am CF-Zentrum.

Einmal im Monat findet eine Fortbildungsveranstaltung speziell zur Mukoviszidose statt.

\section{Jährliche Therapieplanungskonferenz}

Alle am Zentrum betreuten Patienten werden einmal im Jahr im Rahmen der Therapie-Planungskonferenz umfassend diskutiert. Diese Konferenz im Februar dauert zweimal drei Tage und es nehmen alle Mitglieder des Behandlungsteams teil. Nach einer klar definierten Abfolge werden zu jedem Patienten alle relevanten Fakten präsentiert. Dabei sind die Auswertungen und grafischen Darstellungen aus der Datenbank eine wichtige Unterstützung. Besondere Beachtung findet der Verlauf der letzten Jahre, vor allem hinsichtlich Lungenfunktion, Entzündungsparametern und Körpergewicht. Jede Berufsgruppe erarbeitet daraufhin separat ein Ziel für den jeweiligen Patienten. Anschließend wird im Team ein gemeinsamer Therapieplan entworfen, der alle Behandlungsmaßnahmen enthält. Diese Vorschläge werden im Rahmen der „Block“-Sprechstunde im Mai mit dem Patienten besprochen und gegebenenfalls modifiziert (siehe unten).

\section{Qualitätsmanagement wird im Alltag praktiziert}

Wie schon erwähnt, ist das Zentrum seit 2006 ISO-zertifiziert. Voraussetzung dafür war, dass alle Behandlungsabläufe sorgfältig konzipiert und schriftlich festgelegt wurden. Seither wird das Vorgehen fortlaufend weiter optimiert mit dem Ziel, die Qualität kontinuierlich weiter zu verbessern ( $\bullet$ Tab. 2).

Auch für die Nachverfolgung innerhalb des Teams vereinbarter Maßnahmen wird gesorgt. Mitarbeiter nutzen Checklisten, um zu dokumentieren, dass sie bestimmte Gespräche geführt oder Behandlungsmaßnahmen durchgeführt haben. Bei den Ambulanzbesuchen wird nachverfolgt, in welchem Ausmaß der Patient vereinbarte Therapieziele erreicht hat und ob eventuell Nachjustierungen nötig sind. 


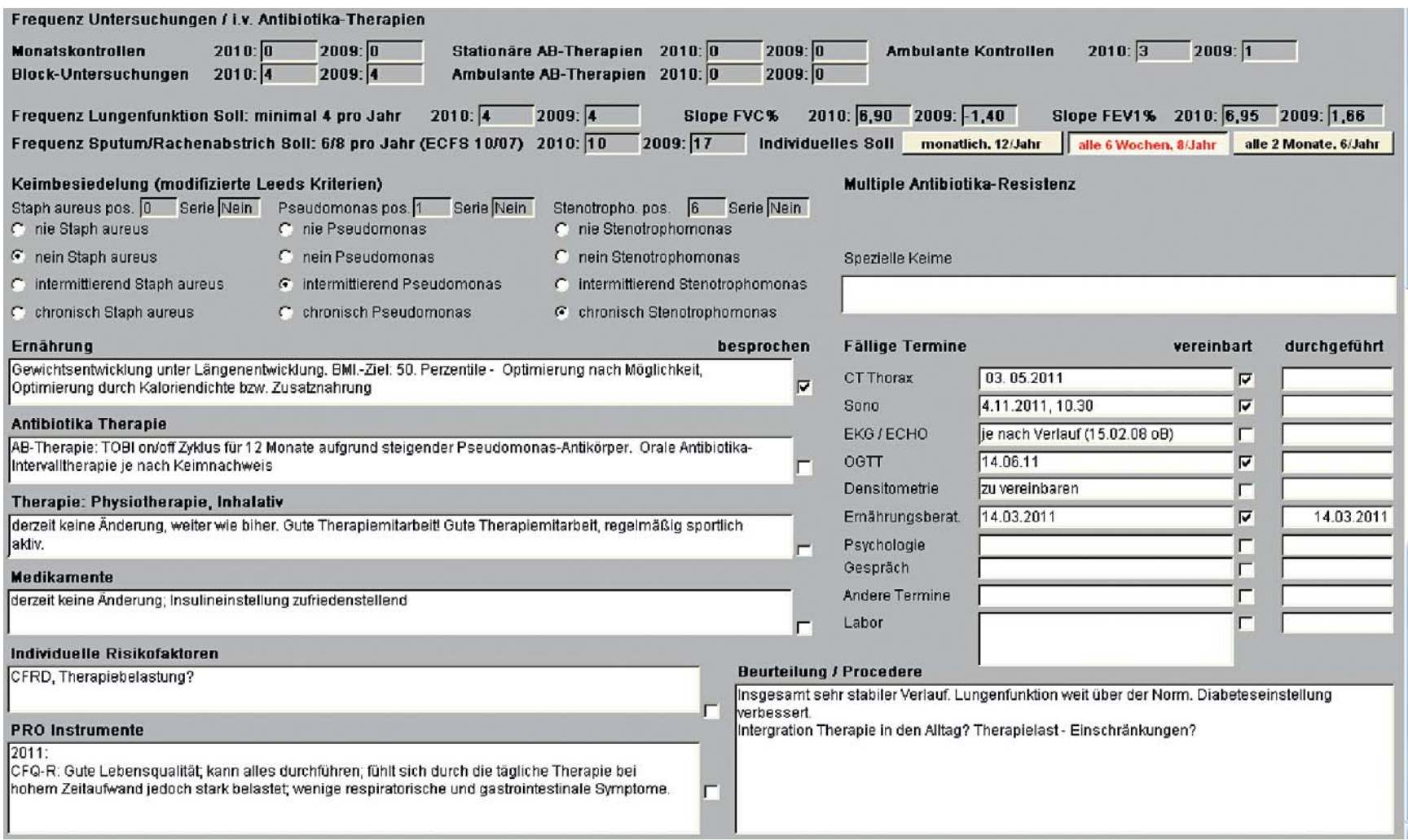

Abb. 1 Tabellarisch aufbereitete Informationen aus der Patientendatenbank zum Verlauf eines Patienten. Während des Ambulanzbesuchs bilden diese Bildschirmmasken die Grundlage für das Arzt-Patienten-Gespräch ( $\bullet$ Tab. 2). Berechnet wird auch die prozentuale Veränderung der FEV1 im vorangegangenen Jahr („Slope FEV1\%“, oben rechts in der Abbildung).

Die eigenen Behandlungsergebnisse werden fortlaufend evaluiert

Mithilfe der Patientendatenbank lassen sich die Behandlungsergebnisse für bestimmte Patientenkohorten über mehrere Kalenderjahre darstellen. Dies ermöglicht den Zentrumsmitarbeitern eine Übersicht über die betreuten Patienten und eine Erfolgskontrolle der eigenen Arbeit.

Neu eingeführte Behandlungsregime werden fortlaufend hinsichtlich des Nutzens für die gesamte Patientengruppe evaluiert. So wird beispielsweise ausgewertet, wie sich die Inhalation mit hypertoner Kochsalzlösung auf die Lungenfunktion der Betroffenen ausgewirkt hat.

Pseudonymisierte Patientendaten werden außerdem an Patientenregister in Deutschland und Österreich übergeben. Der ärztliche Leiter arbeitet aktiv am Projekt „Benchmarking“ des Mukoviszidose e.V. in Bonn mit. Die daran teilnehmenden $26 \mathrm{Ambu}-$ lanzen werten ihre Behandlungsergebnisse systematisch aus und vergleichen sie miteinander. Bei mehreren Ergebniskriterien nimmt die Ambulanz aus Innsbruck im Vergleich zu anderen Einrichtungen Spitzenpositionen ein (siehe $\bullet$ Abb. 4).

\section{Personelle Ausstattung}

$\nabla$

\section{Multiprofessionelles Behandler-Team}

Die personelle Aufstellung orientiert sich an den Europäischen Standards der CF-Versorgung [5] und ist in $\bullet$ Tab. 3 zusammengefasst.

Der ärztliche Leiter des CF-Zentrums ist pädiatrischer Gastroenterologe. Er arbeitet zu etwa 70\% der Arbeitszeit für Mukoviszidose. Ein weiterer in CF spezialisierter Facharzt steht mit rund
Tab.3 Das multidisziplinäre Behandlungsteam nach den Europäischen Standards der Mukoviszidose-Versorgung [5] und ihre Umsetzung in Innsbruck.

\begin{tabular}{|c|c|}
\hline Personal & $\begin{array}{l}\text { Teammitglied } \\
\text { in Innsbruck }\end{array}$ \\
\hline Zentrumsleiter & + \\
\hline Ärztliche CF-Spezialistln & + \\
\hline CF-Pflegekraft & + \\
\hline Physiotherapeutln & + \\
\hline Diätassistentln/ Diätologln & + \\
\hline Sozialarbeiterln & E \\
\hline Psychologln & + \\
\hline Apothekerln & $\mathrm{E}$ \\
\hline Klinische Mikrobiologln & $\mathrm{E}$ \\
\hline \multicolumn{2}{|c|}{$\begin{array}{l}\text { +: Mitglied des multidisziplinären CF-Teams } \\
\text { E: Mitglied des erweiterten Teams, d. h. Personal aus dem Klinikum wird be } \\
\text { Bedarf oder zur Konsultation herangezogen } \\
\text { Kooperationen bestehen zusätzlich mit fixen Ansprechpartnern der Inns- } \\
\text { brucker Universitätskliniken für Radiologie I, Gynäkologie und Geburtshilfe } \\
\text { Hör-, Stimm- und Sprachstörungen, Visceral-, Transplantations- und Thorax } \\
\text { chirurgie, Herzchirurgie, Innere Medizin IV (Nephrologie und Hypertensio- } \\
\text { logie) und Innere Medizin II (Gastroenterologie und Hepatologie). }\end{array}$} \\
\hline
\end{tabular}

$80 \%$ seiner Arbeitszeit für CF-Patienten zur Verfügung. Hinzu kommt eine Assistenzärztin in Ausbildung zur Kinder- und Jugendärztin, die auf einer Viertelstelle mitarbeitet.

Im ambulanten Bereich sind zwei CF-Schwestern tätig, die gemeinsam die Sprechstunden abdecken. Zu ihrer Finanzierung müssen Drittmittel herangezogen werden.

An der Klinik stehen vier Physiotherapeutinnen auf 2,25 Planstellen für CF-Patienten zur Verfügung. Zusätzlich arbeiten zwei niedergelassene Physiotherapeutinnen mit Mukoviszidosepatienten. 
Zum Team gehören zwei Diätologinnen auf 0,25 Planstellen, die sowohl für die ambulante als auch für die stationäre Betreuung zuständig sind. Zu Ärzten und Physiotherapeutinnen besteht ein enger Kontakt mit regelmäßigem Austausch.

Eine Psychologin mit 25\% Arbeitsanfall für Mukoviszidose gehört zum engen CF-Team. Sie nimmt an allen Besprechungen teil und ist während der Block-Sprechstunden direkt vor Ort verfügbar. Alle Patienten kennen die Psychologin und viele Betroffene nehmen auch direkt mit ihr Kontakt auf, wenn Beratungsbedarf besteht. Die Psychologin arbeitet ansonsten in externen Räumlichkeiten im Bereich Univ.-Klinik für Medizinische Psychologie und kann dort auch Patienten separat psychotherapeutisch betreuen. Ein wichtiger Erfolgsfaktor ist die Konstanz der Behandler, die meist schon viele Jahre zum CF-Team gehören und konstruktiv und motiviert mitarbeiten.

\section{Weitere Mitarbeiter}

In der medizinischen Mikrobiologie kümmern sich zwei Ärzte speziell um die Befunde von Mukoviszidosepatienten, inklusive Genotypisierung der isolierten P. aeruginosa-Stämme. Diese Mikrobiologen nehmen an einer Besprechung teil, die gemeinsam mit den CF-Ärzten alle sechs Wochen stattfindet. Sie beinhaltet sowohl die Diskussion einzelner Patienten als auch die krankenhaushygienische Situation.

Ein reger Gedankenaustausch besteht mit dem Leiter der Klinikapotheke. Er sorgt dafür, dass spezielle Medikamente zur Verfügung gestellt werden können, wie beispielsweise neue inhalative Antibiotika. Wenn eine ambulante i.v.-Therapie organisiert werden muss, arbeitet der Klinikapotheker mit den örtlichen Apothekern zusammen und unterstützt sie bei der Bereitstellung der Antibiotika.

Im Unterschied zu vielen anderen CF-Ambulanzen gibt es keinen Sozialarbeiter im engen Behandler-Team. Allerdings stehen die Sozialarbeiterinnen des Departments für Kinder- und Jugendheilkunde unterstützend zur Verfügung, beispielsweise bei Anträgen auf Pflegebeihilfe oder bei Fragen zur zukünftigen beruflichen Orientierung von Jugendlichen. Der Mukoviszidose-Elternverein übernimmt ebenfalls einige Arbeiten aus diesem Bereich, beispielsweise wenn es um Anschaffung von Zusatznahrungen und diesbezügliche Verhandlungen mit den Kassen geht.

Eine sportmedizinische Beratung ist im Aufbau. Mit dem Institut für Sport-, Alpinmedizin und Gesundheitstourismus (IASG) an der Universität Innsbruck arbeitet man eng zusammen, zumal erwachsene Patienten dort spezielle Lungenfunktionsuntersuchungen erhalten.

\section{Diagnostische Aspekte}

\section{Lungenfunktionsdiagnostik}

Die Qualität der Lungenfunktionsuntersuchung ist entscheidend von den Mitarbeitern abhängig. Dies zeigte sich, als nach einem Personalwechsel sogar stabile Patienten schlechtere Werte aufwiesen. Als Referenzwerte werden in Innsbruck die Normwerte nach Knudson [6] herangezogen. Sie haben den Vorteil fortlaufender Sollwertkurven über die Altersgruppen Schulkinder, Jugendliche und Erwachsene. Verwendet man dagegen für Kinder und Jugendliche Normwerte nach Zapletal [7] und für Erwachsene nach Quanjer [8], kommt es mit Erreichen des 18. Lebensjahres zu einem abrupten Abfall der Messwerte, bezogen auf den Sollwert um bis zu $10 \%$ für die FEV1.
Für die Bewertung der Lungenfunktionsergebnisse spielt der Verlauf eine entscheidende Rolle. Dieser wird grafisch auf dem Bildschirm dargestellt. Zusätzlich liefert das Datenbanksystem für jeden Patienten den individuellen mittleren Abfall (Slope) der FEV1 und der MEF25 während der vorausgegangenen 12 Monate. Dieses zahlenmäßige Ergebnis wird mit den üblichen Werten für das Alter des Patienten verglichen und geht ebenfalls in die Beurteilung der aktuellen Situation ein.

\section{Multiple Breath Washout Technik}

Zusätzlich zu Spirometrie und Bodyplethysmografie wurde die Multiple Breath Washout Technik eingeführt und wird den Patienten zur Messung des Lung Clearance Index (LCI) in Kürze routinemäßig zur Verfügung stehen. Sie hat sich als wertvoll für Patienten mit normaler FEV1 erwiesen: Auch wenn die Spirometrie noch weitgehend normale Resultate ergibt, kann der LCI bereits pathologische Werte aufweisen und eine Lungenschädigung anzeigen [9].

\section{Computertomografie}

Seit 1993 wird einmal im Jahr eine thorakale Computertomografie durchgeführt und zwar als Multidetektor-CT mit einer speziellen Ultra-low-dose-Technik [9]. Die resultierende Strahlenbelastung ist mit 0,15 mSv kaum höher als die einer Röntgen-Thoraxaufnahme in zwei Ebenen, auf die in der Routineversorgung verzichtet wird. Das CT wird mithilfe eines Punktesystems befundet und liefert wertvolle Angaben zur Lungenmorphologie, auch bei Patienten mit normaler Spirometrie.

\section{Oraler Glukosetoleranztest (oGT)}

Orale Glukosetoleranztests werden bei Patienten ab 10 Jahren regelmäßig einmal im Jahr durchgeführt, mit Ausnahme der pankreassuffizienten Patienten. Ist der oGT positiv, wird er spätestens nach sechs Monaten wiederholt. Ein CF-Diabetes wird auf diese Weise früh erkannt und mit Insulin therapiert. Zusätzlich zu den Routineterminen werden oGTs auch bei Patienten mit Hepatopathie häufiger durchgeführt oder wenn ein Abfall der FEV1 verzeichnet wurde.

\section{Patientenorientierte Behandlungsergebnisse}

Seit mehreren Jahren werden Behandlungsergebnisse auch aus Sicht der Patienten erhoben. Sie erfassen systematisch einmal im Jahr etwaige Belastungen von Patient und Familie durch bedeutsame Ereignisse. Die Diagnostik zu diesen „Patient reported outcomes“ (PROs) erfolgt durch die Psychologin und ihre Mitarbeiter. Auf einem Tablet-PC tragen die Patienten nach Anleitung durch eine Praktikantin ihre Antworten in den elektronischen Fragebogen ein, wobei im Ambulanzablauf 15 Minuten fürs Ausfüllen reserviert sind. Verwendet werden eine Problemcheckliste (Cystic Fibrosis Problem Checklist [10]), Fragebögen zur krankheitsspezifischen Lebensqualität für die verschiedenen Altersgruppen (CFQ-R [11]) sowie die „Life Event Scale“ des Lebensqualitätsfragebogens aus dem Karolinska Hospital [12].

\section{Therapeutische Aspekte}

\section{$\nabla$}

\section{Physiotherapie}

Die Physiotherapie spielt eine besonders wichtige Rolle in der Betreuung. Die Patienten erlernen individualisiert altersentsprechende physiotherapeutische Techniken, wie die Autogene Drainage. Trainiert werden auch die optimale Inhalationstechnik und 
die Verwendung eines PEP-Systems. Die Vorstellung bei der Physiotherapeutin erfolgt alle sechs Wochen und im Rahmen des vierteljährlichen Ambulanzbesuchs und einmal zwischendurch. Säuglinge und Kleinkinder unter zwei Jahren werden einmal pro Monat in der Ambulanz gesehen und stellen sich dann auch bei der Physiotherapie vor. Die beiden niedergelassenen Krankengymnastinnen, die in die Betreuung eingebunden sind, arbeiten eng mit den Klinik-Physiotherapeutinnen zusammen und erstatten regelmäßig Bericht.

Bei stationär aufgenommenen Patienten geben die Physiotherapeutinnen außerdem eine Rückmeldung über den Behandlungserfolg. Stellen sie beispielsweise fest, dass eine neue AntibiotikaKombination keine Verbesserung für den Patienten bringt, informieren sie die behandelnden Ärzte darüber. Es besteht insgesamt ein reger Gedankenaustausch zwischen Ärzten und Physiotherapeutinnen.

\section{Ernährungsberatung}

Die Ernährungsberatung ist integraler Bestandteil der Versorgung. Alle Teammitglieder haben Zugriff auf die Ernährungsdaten jedes Patienten. Bei der jährlichen mehrtägigen Therapieplanungssitzung wird für jeden Patienten festgelegt, wann er die Ernährungsberaterin sehen soll (sofort, während der nächsten 6 Monate oder bei der Jahresuntersuchung).

Behandlungsziel für jeden Patienten ist, die 50er Perzentile des Body-Mass-Index zu erreichen oder zu übertreffen. Der Verlauf der Body-Mass-Index-Perzentile wird bei den vierteljährlichen Kontrolluntersuchungen beurteilt. Abweichungen von der gewünschten Entwicklung führen zu diagnostischen und therapeutischen Konsequenzen.

Ähnlich wie für die Lungenfunktion feste Zeiten eingeplant sind, nehmen die Patienten im Rahmen der vierteljährlichen, routinemäßigen Block-Untersuchungen Termine bei der Ernährungsberatung wahr, gegebenenfalls auch häufiger. Darüber werden die anderen Teammitglieder vorab informiert.

Auch die Physiotherapeutinnen schauen sich regelmäßig die Daten der Patienten an, die für sie einbestellt sind. Wenn Auffälligkeiten beim BMI bestehen, nimmt die Physiotherapeutin mit der Ernährungsberatung und dem Arzt Kontakt auf und vereinbart einen Beratungstermin für den Patienten.

\section{Strukturierte Patientengespräche}

Für bestimmte Lebenssituationen sind Gespräche zwischen Arzt, Psychologin und Betroffenen vorgesehen. Dazu wurden Gesprächsleitfäden entwickelt. Ein Beispiel ist das „Jugend-Gespräch“, das zum Ziel hat, dem Heranwachsenden die Diagnose Mukoviszidose gewissermaßen neu zu vermitteln und mit ihm die Konsequenzen für seine Lebensperspektive zu diskutieren. Ein „Partner-Gespräch“ wird angeboten, wenn die Patienten einen festen Lebenspartner haben und Themen wie Fertilität und Kinderwunsch besprechen möchten.

Informationen aus diesen Gesprächen werden grundsätzlich vertraulich behandelt und gelangen auch nicht in die Datenbank. Lediglich ein Extrakt dessen, was für die weitere Betreuung erforderlich ist, wird den anderen Mitarbeitern zugänglich gemacht.

\section{Stationäre Versorgung}

Durch eine neuartige Organisation innerhalb der Kinderklinik hat sich für Kinder und Jugendliche im stationären Bereich vieles verändert. Die bisherige Zuordnung zu den Stationen wurde aufgehoben und die Schwesternteams wurden vollkommen neu gebildet. Dies ist eine Herausforderung für die spezialisierten $\mathrm{CF}$ -

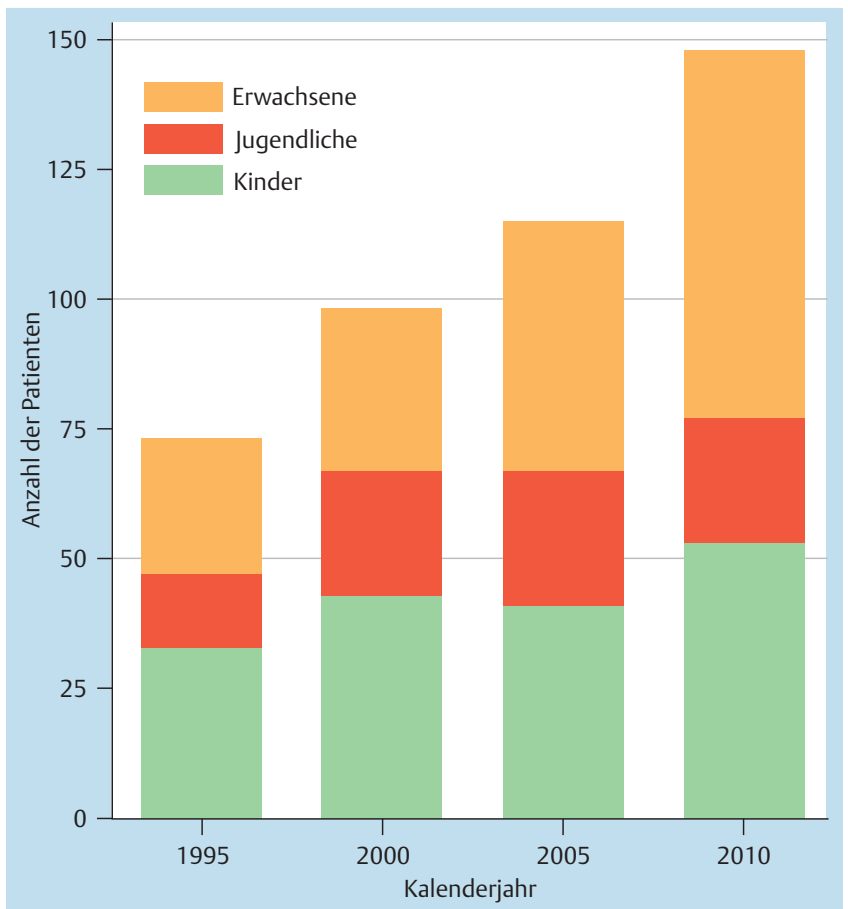

Abb. 2 Anstieg der Patientenzahlen am CF-Zentrum Innsbruck innerhalb von 15 Jahren.

Schwestern, die ihre teilweise neuen Kolleginnen auf den Krankenstationen trainieren und unterstützen müssen.

Erwachsene Patienten werden an der Abteilung für Innere Medizin aufgenommen

\section{Ergebnisse der Versorgung}

Die Patientenkohorte der Innsbrucker Ambulanz umfasst aktuell 148 Patienten, davon 53 Kinder bis 11 Jahre, 24 Jugendliche (12 bis 17 Jahre) und 71 Erwachsene. Der älteste Patient ist 56 Jahre alt und wird seit 10 Jahren in Innsbruck betreut. Die Patientenzahl hat in den letzten 15 Jahren deutlich zugenommen (siehe Abb. 2). Bei den Erwachsenen kam es fast zu einer Verdreifachung (von 26 auf 71 Patienten), sodass in 2010 knapp die Hälfte der Patienten 18 Jahre und älter war.

Eine normale Pankreasfunktion hatten 4 Patienten unter 18 Jahren sowie 7 erwachsene Patienten, einen CF-Diabetes 1 bzw. 13 Patienten dieser beiden Altergruppen. Chronisch bzw. intermittierend mit $P$. aeruginosa kolonisiert waren 6,5\% bzw. 15,6\% der Kinder und Jugendlichen sowie 38,0\% bzw. 18,3\% der Erwachsenen mit CF.

In den Jahren 2009 und 2010 wurden 6 Patienten neu diagnostiziert. Vier Patienten wurden im fortgeschrittenen Krankheitsstadium bzw. zur Transplantations-Listung von anderen Zentren übernommen. In 2010 verzogen 2 Patienten (nach 3 bzw. 6 Jahren Betreuung) in andere Regionen. Zwischen 1995 und 2010 verstarben 16 Patienten des Zentrums Innsbruck sowie 3 Patienten, die im Terminalstadium von auswärts zugewiesen worden waren.

In Innsbruck werden auch Lungen- und Lebertransplantationen durchgeführt, sodass auch Patienten aus anderen Zentren zur Evaluation vorgestellt werden. Derzeit sind für jede der beiden Organtransplantationen jeweils 3 Patienten gelistet. 


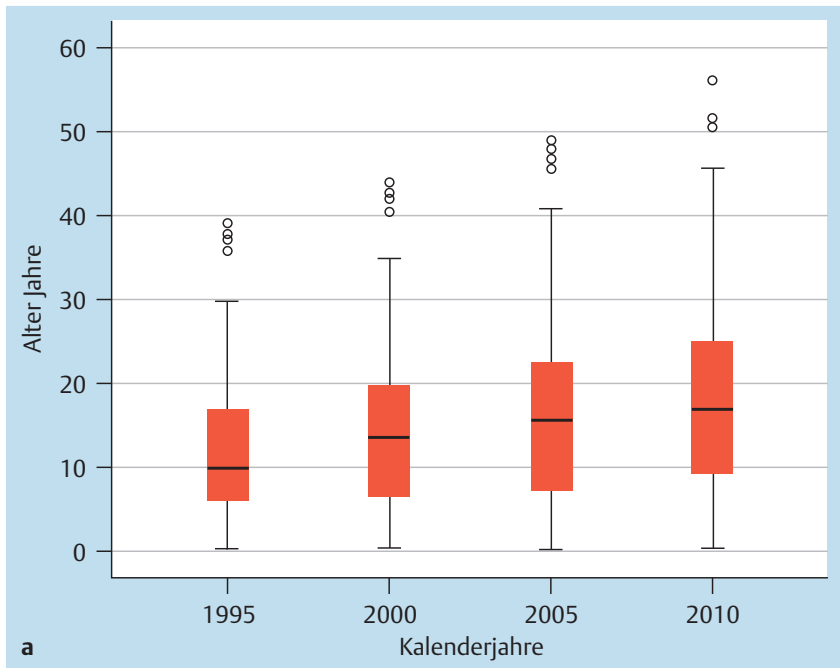

Abb. 3a Boxplots des Alters der Patienten von 1995 bis 2010. Medianes und mittleres Alter stiegen über 15 Jahre an.

\section{Bessere Gesundheit im Vergleich zu Vorjahren}

In $\odot$ Abb. $\mathbf{3}$ a u. b wird am Beispiel der FEV1 dargestellt, wie sich die Mittelwerte der Patientenkohorten in den letzten beiden Jahrzehnten entwickelt haben. Betrachtet man die Werte aller Patienten, die eine Lungenfunktionsprüfung absolvieren konnten (in der Regel ab einem Alter von 5 Jahren), so hat das durchschnittliche Alter der behandelten Patienten von 1995 bis 2010 zugenommen. Bei der FEV1 stellt man im selben Zeitraum eine stabile Situation bzw. einen leichten Anstieg der Mittelwerte fest. Höheres Alter ist üblicherweise mit geringeren FEV1-Werten assoziiert, sodass die Konstanz der mittleren FEV1-Werte als günstige Tendenz zu werten ist. Die Jahresmittelwerte der FVC entwickelten sich ähnlich und stiegen von 82,1\% des Solls in 1995

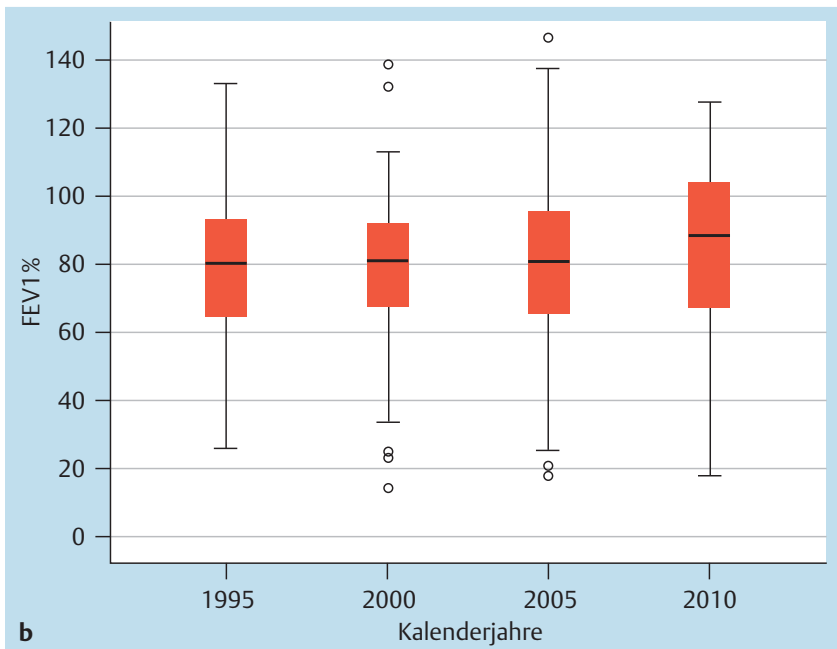

Abb. 3b Boxplots der FEV1-Ergebnisse (in Prozent des Sollwerts) von 1995 bis 2010. Trotz ansteigenden Durchschnittsalters der Patienten blieben die FEV1-Werte der Kohorte stabil.

auf $84,7 \%, 88,8 \%$ und $90,8 \%$ des Solls in den Jahren 2000,2005 und 2010 .

\section{Gute Ergebnisse im Vergleich zu anderen} CF-Ambulanzen

Das vom Mukoviszidose e.V. unterstützte Benchmarking-Projekt erlaubt vergleichende Auswertungen der Behandlungsergebnisse unterschiedlicher Zentren. $\triangle$ Abb. 4 zeigt die Ergebnisse von Innsbruck im Vergleich zu den anderen teilnehmenden Einrichtungen.

Die Daten sind nicht adjustiert, sondern als „rohe“ Mittelwerte dargestellt. Es wird deutlich, dass die Werte der Innsbrucker Patienten im Vergleich zu anderen teilnehmenden Einrichtungen

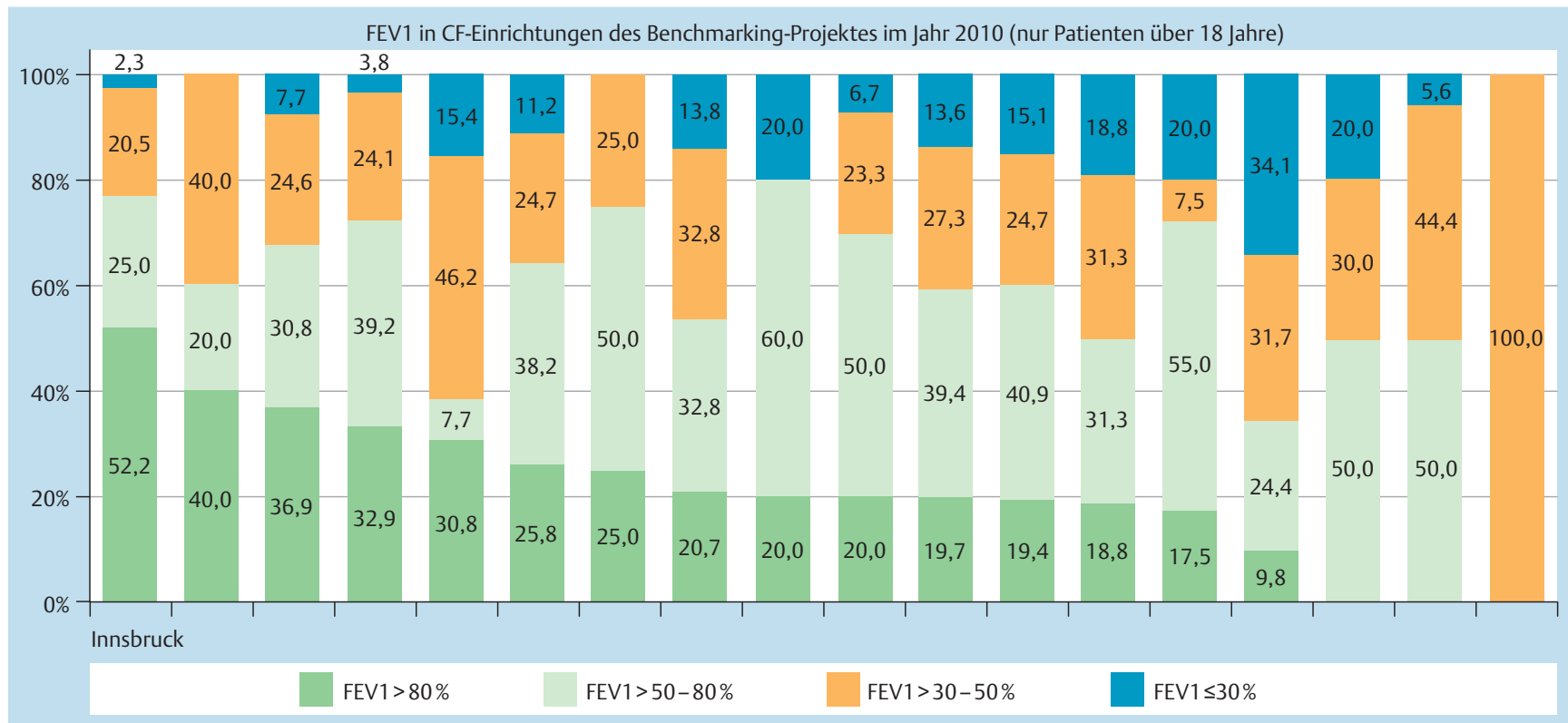

Abb. 4 Verteilung gruppierter FEV1-Werte erwachsener Patienten in den 18 teilnehmenden Behandlungszentren. Jede gestapelte Säule entspricht einem Zentrum und stellt den Anteil der Patienten in den vier FEV1-Kategorien dar. Erwünscht sind große prozentuale Anteile im grünen Bereich, der normalen FEV1-Ergebnissen entspricht (über $80 \%$ des Solls). Die Zahl der pro Zentrum betreuten Patienten liegt zwischen 5 und 119 Personen. Die
54 Erwachsenen aus Innsbruck (linke Säule) hatten den größten Anteil normaler FEV1-Werte (52,3\%), bei 25,0\% lag die FEV1 zwischen 51 und $80 \%$ des Solls, bei 20,5\% der Patienten zwischen 31 und $50 \%$ des Solls und bei einem Patienten (2,3\%) unter $31 \%$ des Solls (mit freundlicher Genehmigung von A. Niemann, Zentrum für Qualität und Management im Gesundheitswesen, Hannover). 
günstig liegen. Die Ergebnisse für Kinder sehen ähnlich erfreulich aus.

\section{Diskussion und Ausblick}

Die multiprofessionelle Behandlung von Mukoviszidosepatienten und ihren Familien an spezialisierten Behandlungseinrichtungen hat sich in Deutschland und Österreich gut etabliert. Damit wurden die von der Europäischen Fachgesellschaft ECFS dargelegten Standards in die Behandlungsroutine integriert. Die Spezialambulanz an der Universität Innsbruck arbeitet in besonderem Maße systematisch und standardisiert, was sich auch in der ISO-Zertifizierung ausdrückt. Die strukturierten Abläufe schaffen Transparenz, fördern die Teamarbeit und tragen nach unserer Auffassung zu den erfreulich guten Behandlungsergebnissen bei, die hier für die Patienten erzielt werden können. Im Vergleich zur Vergangenheit lassen sich die Fortschritte der Gesundheit erkennen, die durch konsequent angewandte pharmakologische Therapie erreicht werden konnten. Die Bereiche Sport, Physiotherapie, Ernährungstherapie und die psychosoziale Betreuung der Betroffenen stellen wichtige Komponenten der multiprofessionellen Versorgung dar. Im Vergleich zu anderen auf Mukoviszidose spezialisierten Behandlungseinrichtungen wurden für die Innsbrucker Patienten gute gesundheitliche Ergebnisse erreicht.

Inwiefern diese Resultate durch das systematisierte und patientenorientierte Arbeiten beeinflusst werden, lässt sich nicht statistisch analysieren und muss daher offen bleiben. Es wurde kein Versuch unternommen, die Behandlungsergebnisse vor und nach Einführung eines strukturierten Vorgehens auf Basis der ECFC-Standards zu evaluieren. Insofern können wir nicht nachweisen, dass der Gesundheitszustand der Patienten durch die Art der Versorgung bedingt ist oder zumindest dadurch mit beeinflusst wird. Es liegt aber auf der Hand, dass strukturierte Abläufe, gut organisierte Ambulanzbesuche und die Verwendung von Checklisten ermöglichen, dass wichtige Diagnostik oder Schulungsgespräche zeitgerecht erfolgen und nicht vergessen werden. Dies ist gerade bei einer komplexen Multiorganerkrankung wie der Mukoviszidose von Bedeutung. Auch tragen die personelle Kontinuität und die enge Zusammenarbeit im Team dazu bei, den Patienten als Person in seinem sozialen Kontext zu erfassen und so die Therapieplanung besser auf die individuellen Möglichkeiten abzustimmen. Die Kooperation mit den Betroffenen während der Sprechstunde ermöglicht, den Patienten als Partner besser einzubeziehen und dürfte seine Therapiebereitschaft fördern.

Die Datenbank mit den Untersuchungsergebnissen steht jedem Teammitglied zur Verfügung. Sie wird vielfältig genutzt, nicht nur zur Patientenversorgung, sondern auch zur Reflexion des eigenen Tuns. So ist die Datenbank integraler Bestandteil der täglichen Arbeit, ohne den eine qualitätvolle Arbeit für Mukoviszidosepatienten nicht mehr denkbar erscheint. In Deutschland wird durch den Mukoviszidose e.V. eine spezielle Dokumentationssoftware zur Verfügung gestellt (MUKO.dok), die ähnliche Zwecke erfüllt. Ihre Vorläufer waren die CFAS-Software und - in den 1990er-Jahren - ein vom Bundesministerium für Forschung gefördertes Projekt zur Entwicklung einer multizentrisch genutzten Mukoviszidose-Datenbank. In Österreich wurde bisher die CFAS-Software verwendet. Eine Umstellung auf MUKO.dok wird nicht erfolgen, sondern die österreichischen Einrichtungen werden sich am Europäischen CF-Register beteiligen. Die Cystic
Fibrosis Foundation der USA hat mit Port CF eine einfache und viel genutzte Software für CF-Patienten entwickelt, die auch von einigen europäischen Ländern übernommen wurde.

Hinsichtlich der Betreuung der inzwischen sehr großen Gruppe der über 18-jährigen Patienten befindet sich das Zentrum in einer Phase des Umbruchs. Als erster Schritt wurde die stationäre Betreuung Erwachsener in der Inneren Medizin eingeführt. Es gibt gemeinsame pädiatrisch-internistische Visiten auf der Station. Der internistische Pneumologe betreut die stationären erwachsenen Patienten mit. Die Therapie-Verantwortung liegt weiterhin beim Leiter des CF-Zentrums. Für die ambulante Betreuung Erwachsener sind gemeinsame Sprechstunden von CFZentrumsleiter und internistischem Pneumologen im CF Zentrum vorgesehen.

Vor kurzem wurde in Innsbruck erstmals eine Patientenbefragung mit einem speziell für CF-Patienten und deren Eltern konzipierten Fragebogen durchgeführt [13]. Damit konnten Versorgungsbereiche identifiziert werden, in denen aus Sicht der Betroffenen noch Verbesserungspotenzial besteht. Änderungen der Gesprächsführung mit jugendlichen Patienten waren eine der ersten Maßnahmen, die daraufhin erfolgten. Patientenzufriedenheitsbefragungen sollen zukünftig etwa alle 2 Jahre durchgeführt werden.

Zusammenfassend erfolgt die multiprofessionelle Arbeit für CFPatienten in Innsbruck klar strukturiert und ISO-zertifiziert. Die medizinische Versorgung basiert auf den Empfehlungen der Europäischen Fachgesellschaft ECFC. Die gesundheitlichen Ergebnisse der Betroffenen haben ein gutes Niveau, auch im Vergleich $\mathrm{zu}$ anderen spezialisierten Einrichtungen. Inwieweit diese Art der Versorgung den Gesundheitszustand der Patienten fördert, kann zwar vermutet, nicht aber wissenschaftlich nachgewiesen werden. Den beschrittenen Weg gehen wir weiter und ergänzen ihn durch neue Elemente, mit dem Ziel, die erreichten Erfolge zu stabilisieren und in einzelnen Bereichen die Arbeit weiter zu verbessern.

\section{Danksagung \\ $\nabla$}

Die Autoren bedanken sich bei allen MitarbeiterInnen des CFTeams für die jahrelange vertrauensvolle Zusammenarbeit: den PhysiotherapeutInnen I. Geissler MSc, A. Nöstlinger, T. Plangger und J. Waldauf, den DiätologInnen V. Jud und I. Wallner sowie bei der Psychologin Dr. U. Smrekar und der Assistensärztin Dr. K. Kovacs.

Dem Elternverein CF TEAM sei Dank für die großzügige Unterstützung der Arbeit am CF-Zentrum.

\section{Interessenkonflikt}

$\nabla$

Die Autoren geben an, dass kein Interessenkonflikt besteht.

\section{Literatur}

1 Buzzetti R, Salvatore D, Baldo E et al. An overview of international literature from cystic fibrosis registries: 1 . Mortality and survival studies in cystic fibrosis. J Cyst Fibros 2009; 8: 229-237

2 Qualitätssicherung Mukoviszidose 2009. Überblick über den Gesundheitszustand der Patienten in Deutschland. Bad Honnef: Hippocampus; 2010

3 Qualitätssicherung Mukoviszidose Österreich - Überblick über den Gesundheitszustand der Patienten in Österreich 2009. Hannover: Zentrum für Qualität und Management im Gesundheitswesen; 2010 
4 Smrekar U, Ellemunter H. Interdisziplinäre Teamarbeit in der Behandlung der zystischen Fibrose: Modell einer psychosomatischen Kooperation. Padiatr Padol 1993; 28: 76

5 Kerem E, Conway S, Elborn S, Heijerman H. Standards of care for patients with cystic fibrosis: a European consensus. J Cyst Fibros 2005; 4: 7-26

6 Knudson RJ, Lebowitz MD, Holberg CJ, Burrows B. Changes in the normal maximal expiratory flow-volume curve with growth and aging. Am Rev Respir Dis 1983; 127: 725 - 734

7 Zapletal A, Samanek M, Paul T. Lung Function in Children and Adolescents. Methods, Reference Values. Basel: Karger; 1987

8 Quanjer PH. Standardized lung function testing. Report Working Party „Standardization of Lung Function Tests“, European Community for Coal and Steel. Bull Europ Physiopathol Respir 1983; 19: 1-95

9 Ellemunter H, Fuchs SI, Unsinn KM et al. Sensitivity of lung clearance index and chest computed tomography in early of lung disease. Respir Med 2010; :
10 Sanders MR, Gravestock FM, Wanstall K, Dunne M. The relationship between children's treatment-related behaviour problems, age and clinical status in cystic fibrosis. J Paediatr Child Health 1991; 27: $290-294$

11 Schmidt A, Wenninger K, Niemann N, Wahn U, Staab D. Health-related quality of life in children with cystic fibrosis: validation of the German CFQ-R. Health Qual Life Outcomes 2009; 7: 97

12 Linde-Edelstam C, Nordlander R, Unden AL et al. Quality-of-life in patients treated with atrioventricular synchronous pacing compared to rate modulated ventricular pacing: a long-term, double-blind, crossover study. Pacing Clin Electrophysiol 1992; 15: 1467-1476

13 Steinkamp G, Stahl K, Ullrich $G$ et al. CF care through the patient's eyes development of a disease specific questionnaire measuring patient satisfaction with CF services. J Cyst Fibros 2010; 9(Suppl 1): 100 